\title{
Osman Tiryaki
}

\section{Method validation for the analysis of pesticide residues in grain by thin-layer chromatography}

Published online: 11 August 2006

(C) Springer-Verlag 2006

The online version of the original article can be found at http://

dx.doi.org.10.1007/s00769-006-0171-3

O. Tiryaki $(\bowtie)$

Turkish Atomic Energy Authority,

Sarayköy Nuclear Research and

Training Center, Application

Department, Agriculture Unit,

Istanbul Road $30 \mathrm{~km}$,

06983 Saray, Ankara, Turkey

e-mail: osmantiryaki@yahoo.com

Tel.: +90-312-8154300

Fax: +90-312-8154307

Regrettably, in Table 2 all values given in the column "Relative residual standard deviation, $S_{\Delta y / \hat{y}}$ " were enlarged by the factor 10 during the production process. The corrected version to Table 2 is as follow.

Table 2

\begin{tabular}{|c|c|c|c|c|}
\hline Analyte $_{\text {detection method }}$ & Linear range, ng $\mu \mathrm{L}^{-1}$ & $\begin{array}{l}\text { Regression equation } \\
y=a+b x\end{array}$ & $\begin{array}{l}\text { Correlation coefficient, } \\
r^{2}\end{array}$ & $\begin{array}{l}\text { Relative residual } \\
\text { standard deviation, } S_{\Delta y / \hat{y}}\end{array}$ \\
\hline Atrazine $_{\mathrm{HILL}}$ & $0.025-0.2$ & $y=5.66+0.657 x$ & 0.969 & 0.043 \\
\hline Chlorpyrifos ${ }_{\mathrm{EBNA}}$ & $0.0125-0.05$ & $y=4.888+1.679 x$ & 0.998 & 0.005 \\
\hline Chlortoluron $_{\text {HILL }}$ & $0.025-0.1$ & $y=5.121+1.512 x$ & 0.999 & 0.006 \\
\hline Cyanazine $_{\text {HILL }}$ & $25-100$ & $y=3.821+0.7214 x$ & 0.996 & 0.006 \\
\hline Dichlorvos $\mathrm{E} \beta \mathrm{NA}$ & $0.5-2$ & $y=3.325+0.056 x$ & 0.996 & 0.012 \\
\hline Dimethoate $_{O-\mathrm{TKI}}$ & $2.5-7.5$ & $y=3.904+0.0067 x$ & 0.999 & 0.004 \\
\hline Dioxacarb $_{O}$-TKI & $0.625-3.125$ & $y=3.7+0.018 x$ & 0.995 & 0.007 \\
\hline Diuron $_{O \text {-TKI }}$ & $0.75-3.75$ & $y=3.821+0.018 x$ & 0.999 & 0.002 \\
\hline Metoxuron $_{\text {HILL }}$ & $0.125-0.5$ & $y=5.624+0.283 x$ & 0.997 & 0.011 \\
\hline Oxamyl $_{O-\mathrm{TKI}}$ & $2.5-12.5$ & $y=4.294+0.01 x$ & 0.996 & 0.011 \\
\hline Oxamyl $_{\text {E } \beta N A}$ & $0.5-2$ & $y=3.138+0.04 x$ & 0.999 & 0.002 \\
\hline Parathion-M $\mathrm{M}_{\mathrm{E} \beta \mathrm{NA}}$ & $0.05-0.2$ & $y=5.792+0.75 x$ & 0.998 & 0.007 \\
\hline Diuron $_{\mathrm{GC}-\mathrm{NPD}}$ & $0.6-1$ & $y=0.084+0.0014 x$ & 0.998 & 0.012 \\
\hline$\underline{\text { Parathion-M }}$ GC-NPD & $0.135-0.5$ & $y=-0.149+0.0124 x$ & 0.999 & 0.029 \\
\hline
\end{tabular}

